\title{
On ionisation effects and abundance ratios in damped Lyman- $\alpha$ systems
}

\author{
D. Schaerer (schaerer@ast.obs-mip.fr) \\ Observatoire Midi-Pyrénées, F-31400 Toulouse, France \\ Y.I.. Izotov (izotov@mao.kiev.ua) \\ Main Astronomical Observatory, Kiev 03680, Ukraine \\ C. Charbonnel (corinne@ast.obs-mip.fr) \\ Observatoire Midi-Pyrénées, F-31400 Toulouse, France
}

\begin{abstract}
The similarity between observed velocity structures of Al III and singly ionised species in damped Ly $\alpha$ systems (DLAs) suggests the presence of ionised gas in the regions where most metal absorption lines are formed. To explore the possible implications of ionisation effects we construct a simplified two-region model for DLAs consisting of an ionisation bounded region with an internal radiation field and a neutral region with a lower metal content. Within this framework we find that ionisation effects are important. If taken into account, the element abundance ratios in DLAs are quite consistent with those observed in Milky Way stars and in metal-poor H II regions in blue compact dwarf galaxies. In particular we cannot exclude the same primary $\mathrm{N}$ origin in both DLAs and metal-poor galaxies. From our models no dust depletion of heavy elements needs to be invoked; little depletion is however not excluded.
\end{abstract}

Keywords: Galaxies, ISM: abundances - Quasars: absorption lines - Intergalactic medium

\section{Introduction}

The study of damped Lyman $\alpha$ systems (DLAs) is of great interest for numerous fields in astrophysics (cf. Petitjean, these proceedings), including nucleosynthesis and the chemical evolution of galaxies and the Universe at large. In addition to investigations on the metallicity evolution with redshift (e.g. Pettini et al. 1994, 1997), spectroscopic studies with $10 \mathrm{~m}$ class telescopes have opened the opportunity to study Ly $\alpha$ absorption lines for a large number of elements including $\mathrm{N}, \mathrm{O}$, $\mathrm{Mg}, \mathrm{Al}, \mathrm{Si}, \mathrm{S}, \mathrm{Cr}, \mathrm{Mn}, \mathrm{Fe}, \mathrm{Ni}$ and $\mathrm{Zn}$.

Two main interpretations of the abundance ratios in DLAs exist currently in the literature - both suffering from several inconsistencies. 1) Lu et al. (1996), Prochaska \& Wolfe (1999), Outram et al. (1999), and Pettini et al. (2000) found that the relative abundance patterns indicate that the bulk of heavy elements in these high-redshift objects were produced by Type II supernovae. However, when combined with the effects of dust depletion (as indicated by the relative overabundance of $\mathrm{Zn}$ relative to $\mathrm{Cr}$ or $\mathrm{Fe}$ ) this interpretation is inconsistent with other

(C) 2018 Kluwer Academic Publishers. Printed in the Netherlands. 
elemental abundance ratios such as $\mathrm{N} / \mathrm{O}, \mathrm{S} / \mathrm{Fe}, \mathrm{Mn} / \mathrm{Fe}$ and $\mathrm{Ti} / \mathrm{Fe}$. This led Lu et al. (1996) and Prochaska \& Wolfe (1999) to conclude that the overabundance of $\mathrm{Zn} / \mathrm{Cr}$ in DLAs may be intrinsic to stellar nucleosynthesis in such systems; a new physical process is then required to explain the damped Ly $\alpha$ abundance patterns. 2) Pettini et al. (1997, 1999), Vladilo (1998) and others argue that the relative abundances of $\mathrm{Cr}, \mathrm{Fe}$, and $\mathrm{Ni}$ are consistent with a moderate degree of dust depletion that, once accounted for, leaves no room for the enhancement of $\alpha$ elements over iron seen in metal-poor stars in the Milky Way. This is in contradiction with a SNII enrichment pattern, but it could be understood if star formation in these systems proceeded at a lower rate than in the early history of our Galaxy (e.g. Pettini et al. 1999; Centurión et al. 2000). A similar conclusion is also drawn from the $\mathrm{Fe} / \mathrm{H}$ vs. redshift distribution of DLAs when compared with our Galaxy (e.g. Lu et al. 1996; Prochaska \& Wolfe 2000).

It is generally believed that the gas in DLAs is mostly in the neutral stage due to the very high optical depth beyond the hydrogen ionisation

limit. Therefore, it is assumed that all species with ionisation potentials lower than hydrogen are in a singly ionised stage, while other species are neutral. However, Lu et al. (1996) and Prochaska \& Wolfe (1999) find a good correlation between the velocity structure of Al III and singly ionised species. The ionisation potential of $\mathrm{Al}^{+}$is $18.8 \mathrm{eV}$, i.e. greater than that of hydrogen. Therefore, $\mathrm{Al}^{+2}$ is likely present in ionised, not in neutral gas. To explain the similarity of Al III and other low ionisation species line profiles, Howk \& Sembach (1999) and Izotov \& Thuan (1999) proposed that these lines originate in the same ionised region or in a mix of neutral and ionised clouds, and stressed the importance of abundance correction for ionisation effects. In the present work we adopt a simple partially ionised two-region model for DLAs and explore the possible implications on abundance ratio determinations within this framework. Full account of this work is given in Izotov et al. (2000, hereafter ISC).

\section{A simplified model}

In the present paper we explore the following "multi-component" picture for DLA systems: 1) an ionisation bounded region illuminated by an internal radiation field complemented by 2) a neutral region with a lower (negligible) metal content. Region 1 is modeled with the ionisation equilibrium code CLOUDY assuming internal stellar radiation and plane-parallel geometry. The outer boundary of the slab is determined by the condition that a low electron temperature of $2000 \mathrm{~K}$ is reached. We adopt stellar ionising spectra with various temperatures $T_{\text {eff }}$, and a 
metal abundance of 0.1 solar with relative element abundances equivalent to those observed in low-metallicity BCDs (e.g., Izotov \& Thuan 1999). The ionisation parameter $U$ is varied over a wide range. Constraints on $U$ are derived from the observed $\mathrm{Al}^{2+} / \mathrm{Si}^{+}$column densities. To explore the average effects, a single "typical" value $(\log U \sim-3)$ is derived from the typical ratio $\log [N(\mathrm{Al}$ III $) / N(\mathrm{Si}$ II $)] \sim-2$ to -2.5 in DLAs. The corresponding ionisation correction factors, which are found to be non-negligible, are applied to all observed abundance ratios to be discussed below (Fig. 2).

A similar model was recently considered by Howk \& Sembach (1999). The main difference with their approach is that they consider ionised density-bounded regions surrounding the neutral, H I-bearing clouds. Both qualitatively and quantitatively the resulting ionisation corrections are quite different from our model. For more details see ISC.

An important feature of both models is that the heavy element abundances in the ionised and neutral regions are a priori unrelated. This implies in particular that relative abundance determinations with respect to hydrogen (e.g. $[\mathrm{Fe} / \mathrm{H}],[\mathrm{Zn} / \mathrm{H}])$, as derived from the observed column densities, may not be meaningful or are at least uncertain.

\section{Implications on abundance ratios in DLAs}

Figure 1 shows that the observed correlation between $[\mathrm{Si} / \mathrm{Fe}]$ and $[\mathrm{Zn} / \mathrm{Fe}]$ which is attributed to progressive dust depletion (Prochaska \& Wolfe 1999) can also be explained by ionisation effects. The best consistency is obtained for the radiation field corresponding to $T_{\text {eff }}=40 \mathrm{kK}$ and $U$ values compatible with the observed $\mathrm{Al}$ III/Si II column density ratios. In this case one finds also that after the ionisation correction is applied (and assuming a final value $[\mathrm{Zn} / \mathrm{Fe}]=0$ ), the resulting $[\mathrm{Si} / \mathrm{Fe}]$ values remain positive (reminiscent of $\alpha$-element enrichment). No additional correction for dust depletion is required, but little depletion is not excluded.

The corrected and uncorrected abundance ratios of various elements are shown in Figure 2 (left). Except for $\mathrm{Cr} / \mathrm{Fe}$, all element ratios with respect to Fe have downward corrections with typical values between $0(\mathrm{Mn} / \mathrm{Fe}),-0.4(\mathrm{Zn} / \mathrm{Fe})$ and $-0.6 \operatorname{dex}(\mathrm{Al} / \mathrm{Fe})$. [With respect to $\mathrm{Zn}$ all ratios except for $\mathrm{Al} / \mathrm{Zn}$ have to be corrected upwards.] After correction we find that $\mathrm{S}$ and $\mathrm{Si}$ (overabundant in $[\mathrm{X} / \mathrm{Fe}]$ ), $\mathrm{Mn}$ and $\mathrm{Al}$ (underabundant), Ti (small overabundance), and $\mathrm{Zn}(\sim$ solar $)$ follow quite well their respective SNII pattern observed in metal-poor halo stars. Cr and Ni (including the oscillator strengths of Fedchak \& Lawler 1999) show some disagreement whose origin is not understood yet (atomic data ? other ?). See ISC for a more detailed discussion. 


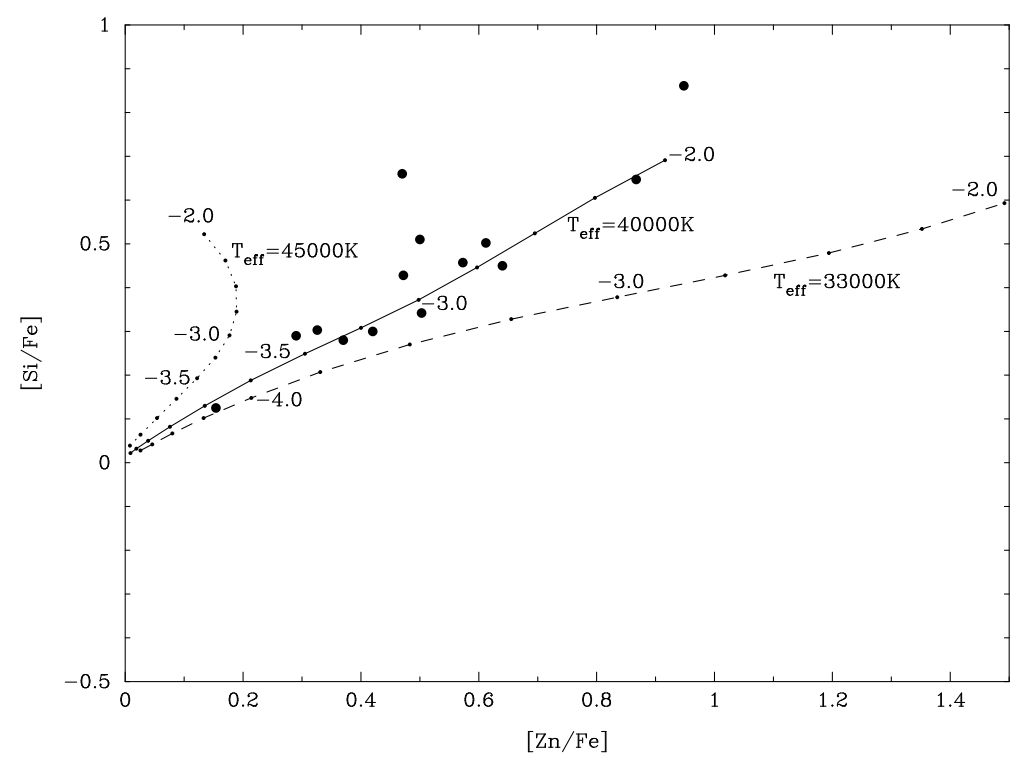

Figure 1. $[\mathrm{Si} / \mathrm{Fe}]$ versus $[\mathrm{Zn} / \mathrm{Fe}]$ showing observations from Prochaska \& Wolfe (1999) and model sequences for different values of $T_{\text {eff }}$ and $U$.
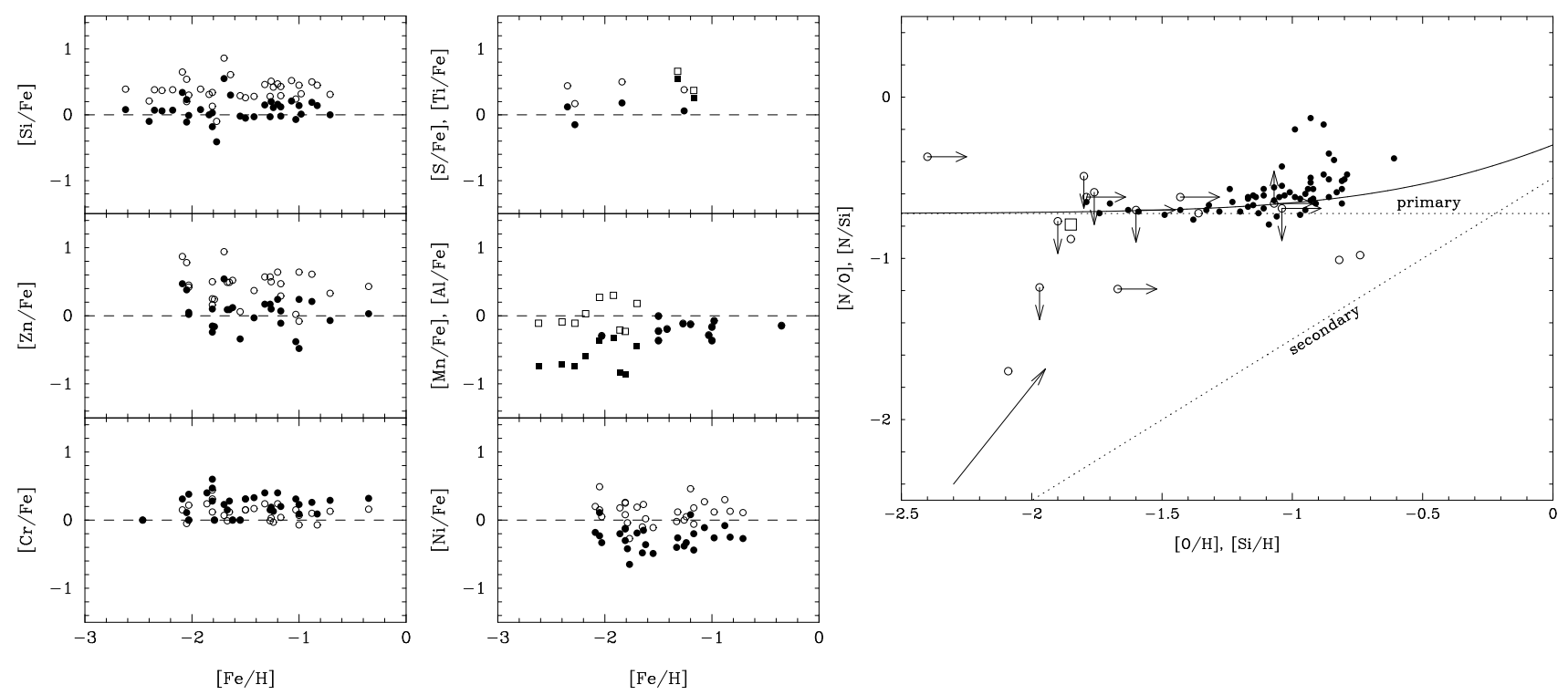

Figure 2. Left: Corrected (filled symbols) and uncorrected (open) abundance ratios based on the data of Lu et al. (1996), Prochaska \& Wolfe (1999) and Pettini et al. (2000) as a function of [Fe/H] (uncorrected). Right: Observed N/O and N/Si ratios in BCDs (filled circles: Izotov \& Thuan 1999) and DLAs (open circles: Lu et al. 1998, Outram et al. 1999) as a function of metallicity. The typical ionisation correction is indicated by the arrow. 
The right panel in Fig. 2 shows observed N/O and N/Si ratios from metal-poor HII regions and measurements in DLAs respectively. From our model we find a typical upward correction of N I/Si II by 0.7 dex, as shown by the arrow. Once accounted for, it appears that the majority of measurements for DLAs are at or above the $\mathrm{N} / \mathrm{O}$ value from the BCDs. This suggests that a significant part of the difference previously found in $[\mathrm{N} / \mathrm{Si}]$ between HII regions and DLAs (Lu et al. 1998) can be explained by ionisation effects. Interestingly, the recent direct measurement of N/O in a DLA from unsaturated OI and NI lines (open square; Molaro et al. 2000), which are also little dependent on ionisation corrections, agrees as well with the metal-poor BCDs. From our analysis we conclude that, if our typical ionisation corrections apply, the present data shows no significant difference in $\mathrm{N} / \mathrm{O}$ abundance ratios between low-metallicity BCDs and DLA systems implying a similar origin of these two elements.

Implications on the metallicity-redshift relation of DLAs and other issues are discussed in ISC.

A detailed discussion of our assumptions and support from other data are presented in ISC. These working hypothesis have been adopted to study the main effects intrinsic stellar ionising radiation may have on observed heavy element patterns in such conditions. Future investigations should hopefully be able to improve on these issues and assess the validity of our picture. It is the hope that the proposed simplified model reflects the main trends due to ionisation effects in DLAs. If correct, our picture offers a clear simplification in the understanding of heavy element abundance ratios in DLAs and their comparison with the local Universe.

\section{References}

Centurión, M., Bonifacio, P., Molaro, P., \& Vladilo, G. 2000, ApJ, 536, 540

Fedchak, J.A., Lawler, J.E. 1999, ApJ, 523, 734

Howk, J. C., \& Sembach, K. R. 1999, ApJ, 523, L141

Izotov, Y.I., Schaerer, D., Charbonnel, C., 2000, ApJ, submitted (ISC)

Izotov, Y.I., Thuan, T.X. 1999, ApJ, 511, 639

Lu, L., Sargent, W. L. W., \& Barlow, T. A. 1998, AJ, 115, 55

Lu, L., Sargent, W. L. W., Barlow, T. A., et al. 1996, ApJS, 107, 475

Molaro, P., Bonifacio, P., et al. 2000, ApJ, in press (astro-ph/0005098

Outram, P. J., Chaffee, F. H., \& Carswell, R. F. 1999, MNRAS, 310, 289

Pettini, M., Ellison, S. L., Steidel, C. C., \& Bowen, D. V. 1999, ApJ, 510, 576

Pettini, M., Ellison, S. L., Steidel, C. C., et al. 2000, ApJ, 532, 65

Pettini, M., King, D. L., Smith, L. J., \& Hunstead, R. W. 1997, ApJ, 478, 536

Pettini, M., Smith, L. J., Hunstead, R. W., \& King, D. L. 1994, ApJ, 426, 79

Prochaska, J., \& Wolfe, A. M. 1999, ApJS, 121, 369

Prochaska, J., \& Wolfe, A. M. 2000, ApJ, 533, L5

Vladilo, G. 1998, ApJ, 493, 583 
contrib_1.tex; $1 / 11 / 2018 ; 13: 44 ;$ p.6 